\title{
Cytogenetic Analysis of Lilium rosthornii
}

\author{
Guangxin Liu \\ College of Biology and the Environment, Co-Innovation Center for the Sustainable Forestry in \\ Southern China, Nanjing Forestry University, Nanjing 210037, China
}

Yue Lan and Haoyang Xin

College of Forestry, Co-Innovation Center for the Sustainable Forestry in Southern China, Nanjing Forestry University, Nanjing 210037, China

Fengrong Hu
College of Landscape Architecture, Nanjing Forestry University, Nanjing, Jiangsu 210037, China

Zhuhua Wu, Jisen Shi, and Mengli Xi ${ }^{1}$

College of Forestry, Co-Innovation Center for the Sustainable Forestry in Southern China, Nanjing

Forestry University, Nanjing 210037, China

\begin{abstract}
Additional Index words. karyotype, chromosome, Giemsa C-banding, propidium iodide banding, 45S rDNA, FISH
Abstract. Lily (Lilium L.) species produce among the most important cut flowers worldwide. China has $\approx 55$ species of Lilium. Although many plants from this genus have been used in hybridization efforts, their cytology has remained unclear. The goal of the current study was to characterize the chromosomes of Lilium rosthornii Diels. Root tips were used to characterize Giemsa C-banding, propidium iodide (PI) banding, and 45S rDNA locations. The karyotype of $L$. rosthornii belongs to type $3 \mathrm{~B}$. C-banding revealed polymorphic banding patterns with the following formula: $2 \mathrm{n}=24=\mathrm{CI}=4 \mathrm{C}+14 \mathrm{CI}+2 \mathrm{I}_{+}+2 \mathrm{I}+2$. Two of the four 45S rDNA hybridization sites were located at pericentromeric positions on the two short arms of the homologues of chromosome 1 , and the other two were located on the long arms of one chromosome 6 homolog and one chromosome 11 homolog. Six of the eight PI bands were detected in the centromeres of the homologues of chromosomes 1,5 , and 8 , and the other two PI bands were detected on the long arms of one chromosome 6 and one chromosome 11. Lilium rosthornii showed enriched banding in both Giemsa C-banding and PI painting. Interestingly, not all 45S rDNA was located in homologous chromosomal locations. These results may provide reference data for $L$. rosthornii for use in further Lilium breeding.
\end{abstract}

Lilium rosthornii is a perennial herb native to China. This Lilium species is an attractive ornamental due to its orange or yellowish flower color, extended floral longevity, curved flower shape, and purple spots in the perianth. With all these desirable flower traits, it is a superior parent for Lilium breeding. Moreover, L. rosthornii is important in traditional Chinese medicine (Long et al., 2004).

Lilium rosthornii was originally primarily distributed in valleys and alongside rivers in provinces such as Sichuan, Chongqing, Hubei, and Guizhou. In recent years, most wild populations of $L$. rosthornii have been reduced due to habitat destruction and loss, and the natural distribution of this species is also shrinking gradually, such that L. rosthornii is becoming endangered (Wang, 2009). However, little is known about the biology and cytology of this species. He (2006) reported on pollen viability and identified the optimal conditions for pollen storage, providing some reference for cross breeding. Zhao (2012) studied the micropropagation and rapid propagation of

Received for publication 18 Mar. 2016. Accepted for publication 13 June 2016. This work was supported by the "948" project of the State Administration of Forestry of China (2013-4-34), Jiangsu Provincial Key Basic Research foundation for Universities (16KJA220001), and a project funded by the Priority Academic Program Development of Jiangsu Higher Education Institutions (PAPD).

We thank Chen Peidu and Wang Xiu'e from Nanjing Agricultural University and to Yuan Shenghua from Yale University for their suggestions and help with the experiments and with writing the paper.

${ }^{1}$ Corresponding author. E-mail: ximenglinjfu@126.com.
L. rosthornii in an attempt to establish the best system for regenerating $L$. rosthornii.

Nonetheless, cytological studies of L. rosthornii remain scarce. Although Qing (2011) and Liu et al. (2010) both analyzed the karyotype of $L$. rosthornii, their results were markedly different. Qing proposed that the karyotype of $L$. rosthornii was type $3 \mathrm{~A}$ [length ratio of the longest chromosome to the shortest chromosome $(\mathrm{Lt} / \mathrm{St})<2: 1$; percentage of chromosomes with an arm ratio of more than $2: 1$ between $51 \%$ and $99 \%$ ] and that chromosomes 1, 7, and 9 were satellite chromosomes, whereas Liu et al. (2010) considered the karyotype of L. rosthornii to be type 3B (Lt/St between $2: 1$ and $4: 1$; percentage of chromosomes with an arm ratio of more than $2: 1$ between $51 \%$ and $99 \%$ ), with chromosomes 1 and 6 constituting satellite chromosomes. Both of those studies used L. rosthornii samples collected from the Jinfoshan area of Nanchuan, Chongqing. The inconsistency between these results complicates the collection, identification, and use of L. rosthornii, as researchers and readers do not know whether this disagreement is caused by differences in the materials themselves or data interpretation. Chromosomal karyotype analysis is mainly based on chromosome number, the arm length ratio, and the locations of secondary constrictions. However, different chromosome preparation methods often cause deviations in the determination of the arm length ratio and location of secondary constrictions (particularly the former). Further studies of this species will provide valuable information for the conservation and utilization of $L$. rosthornii resources. 
Giemsa C-banding and fluorescent in situ hybridization (FISH) techniques are both traditional karyotyping methods. In C-banding analysis, chromosomes are first treated with hydrochloric acid $(\mathrm{HCl})$ and barium hydroxide $\left[\mathrm{Ba}(\mathrm{OH})_{2}\right]$ and then painted with Giemsa stain (Sigma-Aldrich, St. Louis, MO). Different chromosomes typically show different banding patterns that can be used in chromosome identification. In FISH analysis, specific DNA sequences such as ribosomal RNA genes are first labeled with fluorescein, biotin, or digoxin, which can emit visible fluorescence or be detected by visible fluorescence when induced by ultraviolet light. These RNA probes are then hybridized with chromosomes fixed on slides or nylon membranes. The application of FISH to plant chromosome characterization using rDNAs began in the late 1980s and has become a powerful tool for localizing specific DNA sequences on plant chromosomes (Schwarzacher et al., 1989). The rDNAs (45S and 5S), which are repeated sequences in the genome, are commonly used for physical mapping of plant chromosomes due to their universal occurrence and redundancy (Hasterok et al., 2001). Simultaneous FISH of $45 \mathrm{~S}$ and $5 \mathrm{~S}$ rDNAs has been approved as a useful tool for the identification of various plants. Taketa et al. (1999) compared the locations of $5 \mathrm{~S}$ and $18 \mathrm{~S}-25 \mathrm{~S}$ rDNA in nine Hordeum L. species and concluded that rDNA markers are useful for the investigation of chromosome evolution and phylogeny. Mizuochi et al. (2007) first cloned 5S and 45S rDNA from Tulipa fosteriana Hoog and then determined the locations of these two types of rDNA in Tulipa gesneriana L. and T. fosteriana. $5 \mathrm{~S}$ and $45 \mathrm{~S}$ rDNA FISH has also been used to characterize chromosomes from Brassica L. (Hasterok et al., 2001), Cucumis sativus L. cv. Winter Long (Koo et al., 2002), and Nicotiana L. (Lim et al., 2005). Compared with the traditional karyotype analysis, C-banding and FISH provide more uniform and reproducible results. Giemsa C-banding and FISH techniques have been used in the study of Lilium chromosomes (Lee et al., 2014; Sultana et al., 2011). However, to our knowledge, no molecular cytogenetic studies have been performed to date in L. rosthornii.

The PI banding technique was first described by Hanson et al. (1995), who used it with bacterial artificial chromosomes. Peterson et al. (1999) used this technique to determine the location of single-copy and low-copy sequences in tomato (Solanum lycopersicum L). Their results showed that the observation of PI fluorescence revealed bright red fluorescent bands in the nucleolus organizer region (NOR) and in pericentromeric heterochromatin in many chromosomes. More recently, PI fluorochromes have been used in genome mapping using FISH (Nath et al., 2015). In this study, we combined the C-banding, PI-banding, and 45S rDNA FISH methods to characterize the chromosomes from $L$. rosthornii, which was collected in 2004 from Nanchuan, Chongqing Province, China.

\section{Materials and Methods}

Plant material. A sample of L. rosthornii was collected from Nanchuan, Chongqing, China (lat. $29^{\circ} 08^{\prime} 17^{\prime \prime} \mathrm{N}$, long. $107^{\circ} 10^{\prime} 40^{\prime \prime} \mathrm{E}$ ) and was transplanted and propagated in the greenhouse at Nanjing Forestry University, Nanjing, Jiangsu, China. The flower is shown in Fig. 1.

Chromosome preparation. Root tips (1.5-2 $\mathrm{cm}$ in length) were harvested from bulbs at room temperature, pretreated in an ice-water mixture at $4{ }^{\circ} \mathrm{C}$ for $48 \mathrm{~h}$, and fixed in Carnoy's

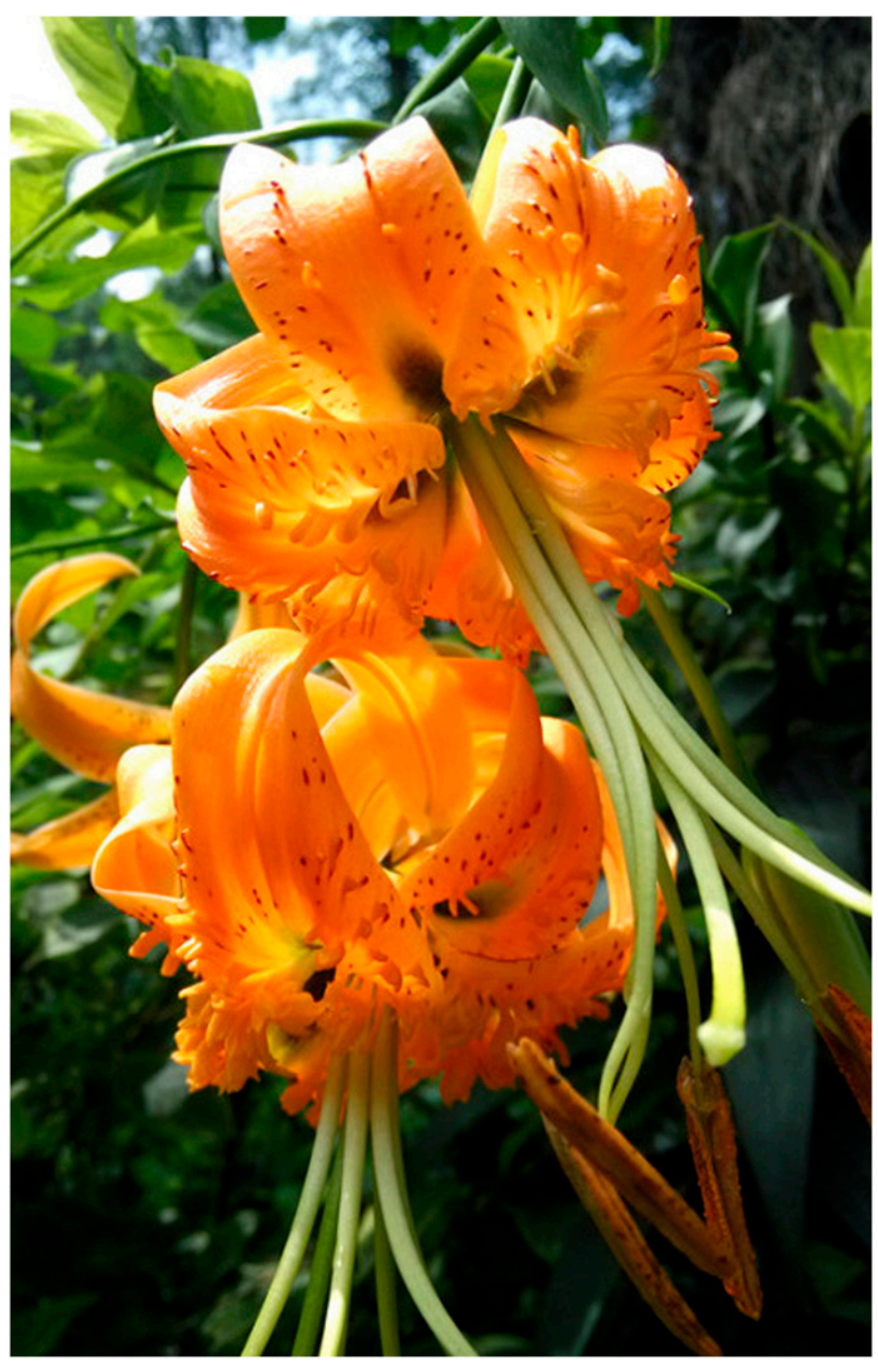

Fig. 1. Flowers of Lilium rosthornii collected from Nanchuan, Chongqing Province, China (lat. $29^{\circ} 08^{\prime} 17^{\prime \prime} \mathrm{N}$, long. $107^{\circ} 10^{\prime} 40^{\prime \prime} \mathrm{E}$ ). The flower characters included rolled petals, yellow or yellow red color, and scattered purplered spots.

fixative (ethanol:acetic acid 3:1, v/v) for $24 \mathrm{~h}$. After washing with distilled water three times, the prepared tissue was stored in $70 \%$ ethanol at $4{ }^{\circ} \mathrm{C}$ until use. For chromosome observation, the fixed root tips were first macerated in $45 \%$ acetic acid for $1.5 \mathrm{~h}$ and then placed on a glass microscope slide. A drop of $45 \%$ acetic acid was added, and the sample was covered with a cover glass and squashed. The slide was dipped in liquid nitrogen for $\approx 20 \mathrm{~s}$. After the slide was removed from the liquid nitrogen, the cover glass was removed with a razor blade. The tissue was then dehydrated in absolute ethanol for $1 \mathrm{~h}$, air-dried, and kept at $-20{ }^{\circ} \mathrm{C}$ until use.

KARYOTYPE ANALYSIS. The karyotype analysis was performed according to $\mathrm{Li}$ and Chen (1985), as shown in Table 1. Karyotyping was performed in accordance with Stebbins (1971), as shown in Table 2.

Giemsa C-Banding analysis. C-banding was used to characterize the chromosomes by labeling their heterochromatin regions. Our procedure was performed following the methods of Gill et al. (1991). Air-dried slides were first immersed in $1 \mathrm{~N}$ $\mathrm{HCl}$ for $1 \mathrm{~min} 10 \mathrm{~s}$ at $60{ }^{\circ} \mathrm{C}$, washed with distilled water for 
$1 \mathrm{~min}$, placed in a saturated $\mathrm{Ba}(\mathrm{OH})_{2}$ solution for $7 \mathrm{~min}$ at room temperature, washed with distilled water for $\approx 5 \mathrm{~min}$, and placed in $2 \times$ saline sodium citrate [SSC (175.5 g NaCl and $88.2 \mathrm{~g}$ trisodium citrate dissolved in a volume of $1000 \mathrm{~mL}$, which was diluted 10-fold with distilled water and then adjusted to $\mathrm{pH} 7.0$ with $10 \mathrm{~m} \mathrm{NaOH}$ before use)] for $1 \mathrm{~h}$ at $60^{\circ} \mathrm{C}$. The slides then were placed in 5\% Giemsa stain for 40-60 min until the desired staining was achieved. C-banding was performed, and photographs were then taken using a microscope with a $63 \times$ objective (DM2500; Leica, Heidelberg, Germany). C-banding analysis was performed according to Li and Zhang (1991).

FLUORESCENT IN SITU HYBRIDIZATION. The slides were prepared as for the C-banding analysis. The cloned 45S rDNA from rice (Oryza sativa L.) was labeled with fluorescein-12dUTP by nick translation. To label the probe, a $50-\mu \mathrm{L}$ labeling mixture containing $5 \mu \mathrm{L} 10 \times$ buffer, $1.5 \mu \mathrm{g} 45 \mathrm{~S}$ rDNA, $5 \mu \mathrm{L}$ dNTP mix (0.4 mM dATP, 0.4 mM dCTP, 0.4 mM dGTP, $0.4 \mathrm{~mm}$ dTTP), 4 U DNA Polymerase I, 0.002 U DNase I, and $1 \mathrm{~nm}$ fluorescein-12-dUTP (Roche, Mannheim, Germany) was reacted at $15^{\circ} \mathrm{C}$ for $2 \mathrm{~h}$ and held at $4{ }^{\circ} \mathrm{C}$ in a polymerase chain reaction instrument (Sultana et al., 2011). Five microliters of ethylenediaminetetraacetic acid $(0.5 \mathrm{M}, \mathrm{pH} 8.0)$ was added to stop the reaction. The FISH procedure was adopted from Lim et al. (2005). First, the chromosomes were pretreated with $100 \mu \mathrm{g} \cdot \mathrm{mL}^{-1}$ DNase-free RNase (Qcbic S\&T, Shanghai, China) dissolved in $2 \times \mathrm{SSC}$ for $1 \mathrm{~h}$ at $37^{\circ} \mathrm{C}$, treated with $5 \mu \mathrm{g} \cdot \mathrm{mL}^{-1}$ pepsin (Amresco, Solon, $\mathrm{OH}$ ) for $10 \mathrm{~min}$ at $37^{\circ} \mathrm{C}$, and fixed in $4 \%$ paraformaldehyde (Sigma-Aldrich) for $10 \mathrm{~min}$ at room temperature. The slides were washed in $2 \times \mathrm{SSC}$ for $5 \mathrm{~min}$, dehydrated in an ethanol dilution series (70\%, 95\%, 100\%), and air-dried. The hybridization mixture consisted of $50 \%$ formamide, $10 \%$ sodium dextran sulfate, $2 \times \mathrm{SSC}, 0.5 \mu \mathrm{L}$ sperm DNA per slide $\left(10 \mathrm{mg} \cdot \mathrm{mL}^{-1}\right)$, and DNA probe (100 ng per slide). The chromosomes were denatured in $70 \%$ formamide at $78{ }^{\circ} \mathrm{C}$ for $1 \mathrm{~min} 10 \mathrm{~s}$, dehydrated in a prechilled $\left(-20{ }^{\circ} \mathrm{C}\right)$ ethanol dilution series $(70 \%, 95 \%, 100 \%)$, and air-dried. The

Table 1. Types of chromosome defined based on centromere location and the arm ratio ( $\mathrm{Li}$ and Chen, 1985).

\begin{tabular}{llc}
\hline Arm ratio $^{\mathrm{z}}$ & Location of centromere & Abbreviation \\
\hline 1 & Median point & $\mathrm{M}$ \\
$1.01-1.70$ & Median region & $\mathrm{m}$ \\
$1.71-3.00$ & Submedian region & $\mathrm{sm}$ \\
$3.01-7.00$ & Subterminal region & $\mathrm{st}$ \\
Above 7.00 & Terminal region & $\mathrm{t}$ \\
$\infty$ & Terminal point & $\mathrm{T}$ \\
\hline
\end{tabular}

${ }^{\mathrm{z}}$ Length of the long arm/length of the short arm.

Table 2. Types of karyotype defined by the ratio of the length of the longest chromosome to that of the shortest chromosome and the percentage of chromosomes with an arm ratio of greater than $2: 1$ (Stebbins, 1971).

\begin{tabular}{lcccc}
\hline & \multicolumn{4}{c}{ Chromosomes with an arm ratio of more than $2: 1(\%)$} \\
\cline { 2 - 5 } $\mathrm{Lt} \mathrm{St}^{\mathrm{z}}$ & 0 & $0.01-0.5$ & $0.51-0.99$ & 1 \\
\hline$<2: 1$ & $1 \mathrm{~A}$ & $2 \mathrm{~A}$ & $3 \mathrm{~A}$ & $4 \mathrm{~A}$ \\
$2: 1-4: 1$ & $1 \mathrm{~B}$ & $2 \mathrm{~B}$ & $3 \mathrm{~B}$ & $4 \mathrm{~B}$ \\
$>4: 1$ & $1 \mathrm{C}$ & $2 \mathrm{C}$ & $3 \mathrm{C}$ & $4 \mathrm{C}$
\end{tabular}

${ }^{\mathrm{z}}$ Ratio of the length of the longest chromosome to that of the shortest chromosome.
DNA probes were denatured at $100{ }^{\circ} \mathrm{C}$ for $10 \mathrm{~min}$ and then cooled on ice. Fifteen microliters of hybridization mix was added to each slide and covered with a cover glass, and the slides were kept in a moist chamber overnight at $37^{\circ} \mathrm{C}$. After hybridization, the slides were washed at $42^{\circ} \mathrm{C}$ in $2 \times \mathrm{SSC}, 50 \%$ formamide, and $2 \times \mathrm{SSC}$ for $10 \mathrm{~min}$ each, followed by a $5 \mathrm{~min}$ wash in $1 \times$ phosphate-buffered saline (PBS). The slides were then counterstained with $5 \mathrm{mg} \cdot \mathrm{mL}^{-1} \mathrm{PI}$ for $4 \mathrm{~min}$, washed three times with $1 \times$ PBS for $\approx 20 \mathrm{~s}$ each time, coated with $10 \mu \mathrm{L}$ mounting medium (VectaShield; Vector Laboratories, Burlingame, CA) and then covered with $20 \mathrm{~mm} \times 20 \mathrm{~mm}$ glass coverslips. Hybridization signals were observed using a fluorescence microscope (BX51; Olympus, Tokyo, Japan). At least 10 metaphase and 10 interphase cells were examined. Selected chromosome spreads and interphase nuclei were photographed with a digital camera (DP72; Olympus).

PI BANDING. The slides used for FISH were counterstained with $5 \mathrm{mg} \cdot \mathrm{mL}^{-1}$ PI and photographed with the DP72 camera.

\section{Results}

The karyotype of $L$. rosthornii based on C-banding was type $3 \mathrm{~B}$, according to Stebbins (1971), and chromosome 2 was a satellite chromosome. The karyotype formula was $2 \mathrm{n}=2 \mathrm{x}=$ $24=4 \mathrm{~m}+12 \mathrm{st}+8 \mathrm{t}$ (Table 3$)$. Lilium rosthornii exhibited polymorphic banding patterns with a formula of $2 n=24=\mathrm{CI}$ (centromere bands and intercalary bands) $=4 \mathrm{C}$ (centromere bands) $+14 \mathrm{CI}_{+}$(centromere and intercalary bands on the long arm) $+2 \mathrm{I}_{+}$(intercalary bands on the long arm) $+2 \mathrm{I}$ (intercalary bands on both long and short arms) +2 (no bands) (Figs. 2, 3A and $\mathrm{B}$ ). Thus, 4 of the 24 chromosomes exhibited centromere bands (C); 14 exhibited both centromere and intercalary bands on the long arm $\left(\mathrm{CI}_{+}\right) ; 2$ only exhibited intercalary bands on the long arm $\left(\mathrm{I}_{+}\right) ; 2$ exhibited intercalary bands on both the long and short arms (I); and 2 exhibited no bands. Band staining in or near the centromere region of a chromosome was always more intense than in other locations. The bands located on chromosomes 1,7 , and 9 were much stronger than those located on the other chromosomes. No distinct band was detected on the short arm of any chromosome except chromosome 8, which had a small band located on the short arm near the centromere. All

Table 3. Relative length (RL), arm ratio (r), and type of each chromosome in five Lilium rosthornii metaphases (Li and Chen, 1985).

\begin{tabular}{lccc}
\hline Chromosome number & $\mathrm{RL} \pm \mathrm{SE}^{\mathrm{z}}$ & $\mathrm{R}^{\mathrm{y}}$ & Nomenclature \\
\hline 1 & $12.04 \pm 0.23$ & 1.59 & $\mathrm{~m}$ \\
$2^{\text {sat }}$ & $10.93 \pm 0.27$ & 1.68 & $\mathrm{~m}$ \\
3 & $10.26 \pm 0.19$ & 9.33 & $\mathrm{t}$ \\
4 & $8.84 \pm 0.10$ & 7.82 & $\mathrm{t}$ \\
5 & $8.31 \pm 0.32$ & 6.38 & $\mathrm{st}$ \\
6 & $8.19 \pm 0.21$ & 8.10 & $\mathrm{t}$ \\
7 & $8.13 \pm 0.13$ & 3.45 & $\mathrm{st}$ \\
8 & $7.19 \pm 0.27$ & 5.42 & $\mathrm{st}$ \\
9 & $7.18 \pm 0.14$ & 3.23 & $\mathrm{st}$ \\
10 & $6.62 \pm 0.28$ & 7.18 & $\mathrm{t}$ \\
11 & $6.36 \pm 0.32$ & 5.00 & $\mathrm{st}$ \\
12 & $5.95 \pm 0.12$ & 3.45 & $\mathrm{st}$
\end{tabular}

$\overline{{ }^{z} \text { (Average length of each chromosome/average sum of the length of all }}$ chromosomes) $\times 100$.

${ }^{\mathrm{y}}$ Length of the long arm/length of the short arm. 

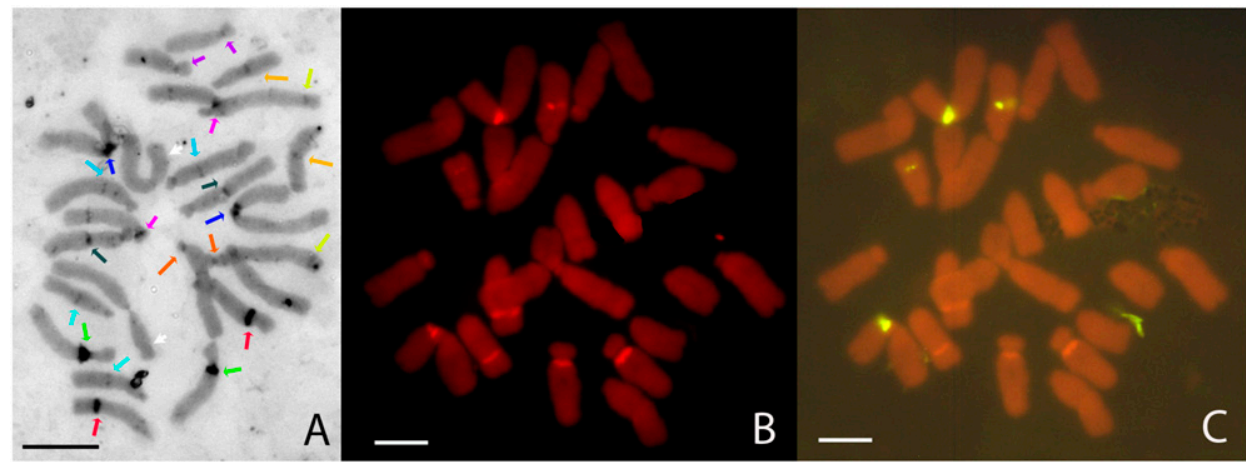

\section{Discussion}

Chromosome characterization is important for karyotyping, cytogenetics, and the physical mapping of genes that control agriculturally important traits in plants (Younis et al., 2015). In this study, we used C-banding, PI-banding, and FISH to characterize the chromosomes of $L$. rosthornii.

Every chromosome could be identified clearly using the combination of these three different methods. The 45S rDNA FISH, PI, and C-banding methods were previously used by Lim et al. (2001) in Lillium longiflorum Thunb and Lillium rubellum Baker, but there are significant differences between our results and those of Lim et al. (2001). In that study, only 11 small bands in L. longiflorum and 12 small bands in L. rubellum, which were minimally effective for characterizing chromosomes, were found. In contrast, the C-banding result in our study showed a typical banding pattern for all of the chromosomes that was similar between homologous chromosomes (the arrow in Fig. 2). Each metaphase chromosome could be identified by C-banding. This difference may be due to the different treatment procedures that we adopted.

PI intercalates between the bases

Fig. 3. Comparisons of homologous chromosomes in Lilium rosthornii using different methods. (A) C-banding karyotype of $L$. rosthornii. Homologues chromosomes showed similar banding patterns, while different patterns were observed between different pairs of homologous chromosomes. (B) C-banding pattern of L. rosthornii. (C) Propium iodide (PI) painting of L. rosthornii. Eight PI bands were detected, at the centromeres of homologous chromosomes pairs 1,5 , and 8 and on the long arms of one chromosome 6 homolog and one chromosome 11 homolog. (D) 45S rDNA fluorescent in situ hybridization analysis of $L$. rosthornii. Two of the four 45S rDNA hybridization sites were located at pericentromeric positions on the short arms of the chromosome 1 homologs, and the other two sites were located on the long arms of one chromosome 6 homolog and one chromosome 11 homolog.

of either single- or double-stranded nucleic acid molecules (HeslopHarrison and Schwarzacher, 1996) and has been used in chromosomal analyses for $\approx 20$ years. However, the only report in which PI staining was used in Lilium species was by Lim et al. (2001). In our research,

chromosomes of L. rosthornii showed unique, specific banding characteristics and could be easily identified. FISH was used to analyze the 45S rRNA gene loci on the somatic chromosomes of $L$. rosthornii in metaphase. Two of the four $45 \mathrm{~S}$ rDNA hybridization sites were located at pericentromeric positions on the short arms of the chromosome 1 homologs, and the other two were located on the long arms of one chromosome 6 homolog and one chromosome 11 homolog (Figs. 2C and 3D).

Eight PI bands were detected on the centromeres of the homologous pairs of chromosomes 1,5 , and 8 and on the long arms of one chromosome 6 and one chromosome 11 (Figs. 2B and $3 \mathrm{C})$. All of the $45 \mathrm{~S}$ rDNA sites showed significant PI banding. The $45 \mathrm{~S}$ rRNA gene loci overlapped with the PI staining sites. However, there were two sites, one on chromosome 5 and one on chromosome 8 that showed significant PI banding without any staining at the $45 \mathrm{~S}$ rRNA loci (Figs. 2B and 3C). we found that eight PI bands are located not only at the same position as the four $45 \mathrm{~S}$ rDNA sites but also in the centromeres of chromosomes 5 and 8 (Figs. 2 and 3). This result is identical to that obtained by Lim et al. (2001). That study showed that PI staining is not confined to the C-banding and $45 \mathrm{~S}$ rRNA gene locations and can provide new markers for chromosome identification. Considering the simple protocol for PI banding and its stability, PI banding may be a useful tool for identifying chromosomes in Lilium and other plant species.

Furthermore, we used $45 \mathrm{~S}$ rDNA as a FISH probe and detected four loci in L. rosthornii; this is the lowest number of $45 \mathrm{~S}$ loci reported in a Lilium species to our knowledge (Hwang et al., 2011; Lee et al., 2014; Lim et al., 2001). Galasso (2003) reported that the number of rDNA loci was reduced under selective pressures, such as the low temperatures that are prevalent in temperate areas. That study also found that during the domestication and evolution of Vigna unguiculata L. Walp 
populations, the number of $45 \mathrm{~S}$ rDNA sites increased. It was also proposed that the increased numbers of $45 \mathrm{~S}$ rDNA clusters may be associated with the diversification and selective pressure on the genus Oryza (Fukui et al., 1994). It is also possible that some rDNA loci could have been lost during evolution (Thomas et al., 1997); in addition, some rDNA loci may presumably have been translocated and combined with other rDNA sequences, as suggested for Brassica (Snowdon et al., 1997). It remains unclear why L. rosthornii shows so few $45 \mathrm{~S}$ rDNA loci. The loci were not all located on homologous chromosomes, which also differ from previous studies in Lilium species (Lim et al., 2001; Sultana et al., 2011). However, the research of Liu et al. (2006) showed that some of the 45S rDNA can also be located individually, but not in pairs, in Salvia miltiorrhiza Bunge and Ulmus pumila L. One possible explanation is that the tandem repeats of rDNA clusters permit chromosomal rearrangements that move the NORs to other chromosome that did not previously have rDNA sequences.

This study has some limitations. First, 45S rDNA was the only FISH probe used in the present study, and only four chromosomes could be detected using this technique. Second, L. rosthornii was the only Lilium species used in this research, and we, therefore, could not compare these results with those of other Lilium species distributed throughout China. Additional Lilium species and FISH probes should be used in subsequent studies.

To the best of our knowledge, no report on the cytogenetic analysis of $L$. rosthornii has been released previously except for rare karyotype analyses (Liu et al., 2010). Traditional karyotyping is mainly based on the length and arm ratio of chromosomes, and there are many technical limitations to those procedures. For example, a chromosome may be too small, or there may be no significant difference in the total length and arm ratio between chromosomes; thus, it is not always possible to unambiguously identify every chromosome. Lilium rosthornii is an important species of wild Lilium that we studied using C-banding, PI banding, and FISH. Rich bands provided sufficient unique characteristics for chromosome identification in L. rosthornii, offering a firm cytological foundation for its further use.

\section{Literature Cited}

Fukui, K., N. Ohmido, and G.S. Khush. 1994. Variability in rDNA loci in the genus Oryza detect through fluorescence in situ hybridization. Theor. Appl. Genet. 87:893-899.

Galasso, I. 2003. Distribution of highly repeated DNA sequences in species of the genus Lens Miller. Genome 46:1118-1124.

Gill, B.S., B. Friebe, and T.R. Endo. 1991. Standard karyotype and nomenclature system for description of chromosome bands and structural aberrations in wheat (Triticum aestivum L.). Genome 34:830-839.

Hanson, R.E., M.S. Zwick, S. Choi, M.N. Islam-Faridi, T.D. McKnight, R.A. Wing, H.J. Price, and D.M. Stelly. 1995. Fluorescent in situ hybridization of a bacterial artificial chromosome. Genome 38:646-651.

Hasterok, R., G. Jenkins, T. Langdon, R.N. Jones, and J. Maluszynska. 2001. Ribosomal DNA is an effective marker of Brassica chromosomes. Theor. Appl. Genet. 103:486-490.

He, L. 2006. Studies on pollen viability and storage conditions of Lilium rosthornii. Trop. Agr. Sci. Technol. 29:13-14.

Heslop-Harrison, J.S. and T. Schwarzacher. 1996. Flow cytometry and chromosome sorting, p. 85-106. In: K. Fukui and S. Nakayama (eds.). Plant chromosomes laboratory methods. CRC Press, Boca Raton, FL.

Hwang, Y.J., H.H. Kim, J.B. Kim, and K.B. Lim. 2011. Karyotype analysis of Lilium tigrinum by FISH. Hort. Environ. Biotechnol. 52:292-297.
Koo, D.H., Y. Hur, D.C. Jin, and J.W. Bang. 2002. Karyotype analysis of a Korean cucumber cultivar (C. sativus L. cv. Winter Long) using $\mathrm{C}$-banding and bicolor fluorescence in situ hybridization. Mol. Cells 13:413-418.

Lee, H., A. Younis, Y.J. Hwang, Y.I. Kang, and K.B. Lim. 2014. Molecular cytogenetic analysis and phylogenetic relationship of $5 \mathrm{~S}$ and $45 \mathrm{~S}$ ribosomal DNA in Sinomartagon Lilium species by fluorescence in situ hybridization (FISH). Hort. Environ. Biotechnol. 55:514-523.

Li, M.X. and R.Y. Chen. 1985. A suggestion on the standardization of karyotype analysis in plants. J. Wuhan Bot. Res. 3:297-302.

Li, M.X. and X.F. Zhang. 1991. Research and technique of plants chromosomes. Northeast For. Univ. Press, Harbin, China.

Lim, K.Y., R. Matyasek, A. Kovarik, J. Fulnecek, and A.R. Leitch. 2005. Molecular cytogenetics and tandem repeat sequence evolution in the allopolyploid Nicotiana rustica compared with diploid progenitors N. paniculata and N. undulata. Cytogenet. Genome Res. 109:298-309. Lim, K.B., J. Wennekes, J.H. de Jong, E. Jacobsen, and J.M. van Tuyl. 2001. Karyotype analysis of L. longiflorum and L. rubellum by chromosome banding and fluorescence in situ hybridization. Genome 44:911-918.

Liu, B., C.B. Chen, X.L. Li, R.Y. Chen, and W.Q. Song. 2006. Physical mapping of $45 \mathrm{~S}$ rDNA on metaphase chromosomes in several plant species. Acta Sci. Natur. Univ. Nankaiensis 39:96-102.

Liu, H.M., L. Zhi, L.H. Zhao, S.Z. Sui, and M.Y. Li. 2010. Karyotype analysis of four wild Lilium species. J. Plant Genet. Resources 11:469-473.

Long, Y.Y., J.Z. Zhang, and L.N. Zhang. 2004. Lily-The king of bulb flower. Jindun Press, Beijing, China.

Mizuochi, H., A. Marasek, and K. Okazaki. 2007. Molecular cloning of Tulipa fosteriana rDNA and subsequent FISH analysis yields cytogenetic organization of $5 \mathrm{~S}$ rDNA and $45 \mathrm{~S}$ rDNA in T. gesneriana and T. fosteriana. Euphytica 155:235-248.

Nath, S., T.B. Jha, S.K. Mallick, and S. Jha. 2015. Karyological relationships in Indian species of Drimia based on fluorescent chromosome banding and nuclear DNA amount. Protoplasma 52:283-299.

Peterson, D.G., N.L.V. Lapitan, and S.M. Stack. 1999. Localization of single- and low-copy sequences on tomato synaptonemal complex spreads using fluorescence in situ hybridization (FISH). Genetics 152:427-493.

Qing, Q.J. 2011. Karyotypes and genetic variations in 9 species of Lilium. MS Diss., Sichuan Agr. Univ., Chengdu, China.

Schwarzacher, T., A.R. Leitch, M.D. Bennett, and J.S. HeslopHarrison. 1989. In situ localization of parental genomes in a wide hybrid. Ann. Bot. (Lond.) 64:315-324.

Snowdon, R.J., W. Kohler, and A. Kohler. 1997. Chromosomal localization and characterization of rDNA loci in the Brassica A and $\mathrm{C}$ genomes. Genome 40:582-587.

Stebbins, G.L. 1971. Chromosomal evolution in higher plants. Edward Arnold, London, UK.

Sultana, S., J.W. Bang, and H.W. Choi. 2011. Organization of the 5S rRNA gene units in Korean Lilium species. Genes Genomics 33:251257.

Taketa, S., G.E. Harrison, and J.S. Heslop-Harrison. 1999. Comparative physical mapping of the $5 \mathrm{~S}$ and $18 \mathrm{~S}-25 \mathrm{~S}$ rDNA in nine wild Hordeum species and cytotypes. Theor. Appl. Genet. 98:1-9.

Thomas, H.M., J.A. Harper, M.R. Meredith, W.G. Morgan, and I.P. King. 1997. Physical mapping of ribosomal DNA sites in Festuca arundinacea and related species by in situ hybridization. Genome 40:406-410.

Wang, R.B. 2009. Studies on conservation biology of Lilium rosthornii Diels, an endemic plant to China. PhD Diss., Southwest Univ., Chongqing, China.

Younis, A., F. Ramzan, Y.J. Hwang, and K.B. Lim. 2015. FISH and GISH: Molecular cytogenetic tools and their application in ornamental plants. Plant Cell Rpt. 34:1477-1488.

Zhao, J. 2012. Study on tissue culture and propagation of Lilium rosthornii. MS Diss., Southwest Univ., Chongqing, China. 